$A B C$ transporters are involved in the eflux of MTX from cells. An increased expression and function of these transporters should decrease MTX concentrations in target cells, resulting in lack of therapeutic response. ABCB1 $3435 \mathrm{C} / \mathrm{T}$ (rs1045642) is a high frequency polymorphism, significantly associated with RA good responses, symptom remission and reduced adverse events, due to MTX treatment (3). Thymidylate synthase (TYMS) is involved in thymidine synthesis. MTX decreases TYMS activity by inhibition and decreasing the access to tetrahydrofolate (THF) cofactors (1). The most common genetic variant of the TYMS gene consists of a $28 \mathrm{bp}$ tandem repeat (rs34743033), with double and triple number of repeats (2R and $3 \mathrm{R})$. The $3 \mathrm{R}$ allele genotype was associated with decreased efficacy and increased toxicity (4).

The 5,10-methylenetetrahydrofolate reductase (MTHFR) enzyme is indirectly inhibited by MTX. The most common SNPs of the MTHFR gene are C677T (rs1801133) and A1298C (rs1801131). Both are associated with a decreased efficacy and an increased toxicity of MTX (5).

Conclusion: MTX response is affected by many gene variants; the effect of each variant separately is likely to be small. Additionally, gene-gene interaction, enhancing the potential role of linkage disequilibrium. This shows the emerging need for a better gene characterization and to improve the knowledge about variants distribution according to ethnicity, to explain different responses to MTX at an individual level. REFERENCES:

[1] Song, G. et al. Association of the MTHFR C677T and A1298C polymorphisms with methotrexate toxicity in rheumatoid arthritis: a meta-analysis. Clin Rheumatol 33, 1715-1724 (2014).

[2] Hayashi $\mathrm{H}$. et al. A single nucleotide polymorphism of reduced folate carrier 1 predicts methotrexate efficacy in Japanese patients with rheumatoid arthritis. Drug Metab Pharmacokinet. 2013;28(2):164-8.

[3] Zhu H. et al. Pharmacogenetics and pharmacogenomics for rheumatoid arthritis responsiveness to methotrexate treatment: the 2013 update. Pharmacogenomics. 2014 Mar;15(4):551-66.

[4] Owen SA. et al. Genetic polymorphisms in key methotrexate pathway genes are associated with response to treatment in rheumatoid arthritis patients. Pharm J. 2013;13:227-34.

[5] Hughes LB. et al. Racial or ethnic differences in allele frequencies of single-nucleotide polymorphisms in the methylenetetrahydrofolate reductase gene and their influence on response to methotrexate in rheumatoid arthritis. Ann Rheum Dis. 2006;65:1213-8.

Disclosure of Interests: None declared

DOI: 10.1136/annrheumdis-2021-eular.3749

\section{AB0015 $\quad$ STUDY OF VDR AND VDBP GENES AS CANDIDATE SUSCEPTIBILITY GENES FOR THE DEVELOPMENT OF IMMUNE-MEDIATED DISEASES IN THE PARAGUAYAN POPULATION}

I. Acosta-Colman ${ }^{1}$, M. Vazquez ${ }^{1}$, S. Cabrera-Villalba ${ }^{1}$, A. Ayala-Lugo ${ }^{2}$, M. E. Acosta ${ }^{2}$, I. Arevalo de Guillen ${ }^{2}$, V. Jolie ${ }^{2}$, M. Duarte ${ }^{1}$, V. Valinotti ${ }^{1}$, R. Contreras ${ }^{3}$, G. Avila ${ }^{1}$, T. Martinez ${ }^{4}$, A. Julià ${ }^{5}$, S. Marsal ${ }^{5} .{ }^{1}$ Hospital de Clinicas, Universidad Nacional de Asuncion, Rheumatology, Asunción, Paraguay; ${ }^{2}$ Insituto de Investigaciones en Ciencias de la Salud, UNA, Asunción, Paraguay; ${ }^{3}$ Hospital de Clinicas, Universidad Nacional de Asuncion, Dermatology, Asunción, Paraguay; ${ }^{4}$ Laboratorio Curie, Laboratorio Curie, Asunción, Paraguay; ${ }^{5}$ Hospital General Vall d'Hebron, Vall d'Hebron Institut de Recerca, Barcelona, Spain

Background: Immune Mediated Inflammatory Diseases (IMIDs) are complex diseases that are believed to have a strong interaction between the genome and the environment as part of their aetiology. In studies using the candidate gene strategy, genetic variation in a gene where functionality has been associated with the pathophysiology of the disease under study is being analyzed. In the last decade, polymorphisms of the vitamin D receptor (VDR) and VDBP genes have been more emphatically studied in IMIDs in different populations, but the results reported have not yet been conclusive.

Objectives: To identify an association between vitamin $D$ receptor (VDR) and vitamin D-binding protein (VDBP) gene polymorphisms, and IMIDs in Paraguayan patients.

Methods: Association study of VDR (SNPs rs731236, rs7975232, rs2228570) and VDBP (rs4588) gene polymorphisms with susceptibility to IMIDs in Paraguayan population. A total of 399 patients with IMIDs (i.e. Systemic Lupus Erythematosus (SLE), Scleroderma (ES), Rheumatoid Arthritis (RA), and Cutaneous Psoriasis (CPS) and 100 hypernormal controls (HC) from the same population were included in this study. Genotyping was performed using Taqman real-time PCR-based technology (Life Technologies, USA). Statistical analysis was performed using Rv3.0.1 statistical language software (www.R-project.org). A p value $\leq 0.05$ was used for statistical significance. Results: A total of 399 individuals, 100 controls and 299 patients (99 RA, 100 SLE, $50 \mathrm{ES}$, and $50 \mathrm{PSO}$ ) were included. Seventy-six percent were female and $24 \%$ were male. The mean age was $43.7 \pm 14$ years. Four SNPs were genotyped: rs731236, rs7975232, rs2228570, rs4588. The HWE test was not statistically significant for any of the 4 SNPs considered $(P>0.05)$, confirming the quality of genotyping and the absence of technical bias. (Table 1).

Table 1. Genotyping of SNPs of the VDR and VDBP gene in Paraguayan population with IMIDs.

\begin{tabular}{lllllllllll} 
SNP & IMID & $\begin{array}{l}\text { Minor Major } \\
\text { Allele }\end{array}$ & $\begin{array}{l}\text { MAlele } \\
\text { Control }\end{array}$ & $\begin{array}{l}\text { MAF } \\
\text { Case }\end{array}$ & OR & IC.L & IC.H & p allelic P.Genetic \\
\hline rs731236 & SLE & G & A & 0.5 & 0.4 & 0.64 & 0.42 & 0.97 & 0.035 & 0.08 \\
rs731236 & RA & G & A & 0.5 & 0.41 & 0.69 & 0.46 & 1.05 & 0.071 & 0.12 \\
rs731236 & SS & G & A & 0.5 & 0.42 & 0.71 & 0.42 & 1.18 & 0.18 & 0.37 \\
rs731236 & GPS & G & A & 0.5 & 0.38 & 0.6 & 0.36 & 1.01 & $\mathbf{0 . 0 4 9}$ & $\mathbf{0 . 0 4 2}$ \\
rs2228570 & SLE & A & G & 0.36 & 0.38 & 1.14 & 0.74 & 1.74 & 0.6 & 0.45 \\
rs2228570 & RA & A & G & 0.36 & 0.31 & 0.83 & 0.53 & 1.28 & 0.4 & 0.56 \\
rs2228570 & SS & A & G & 0.36 & 0.36 & 1.02 & 0.6 & 1.73 & 1 & 0.057 \\
rs2228570 & GPS & A & G & 0.36 & 0.39 & 1.16 & 0.68 & 1.96 & 0.61 & 0.83 \\
rs7975232 & SLE & C & A & 0.36 & 0.32 & 0.82 & 0.53 & 1.26 & 0.4 & 0.072 \\
rs7975232 & RA & C & A & 0.36 & 0.29 & 0.72 & 0.46 & 1.12 & 0.14 & 0.064 \\
rs7975232 & SS & C & A & 0.36 & 0.22 & 0.49 & 0.27 & 0.88 & $\mathbf{0 . 0 1 2}$ & $\mathbf{0 . 0 0 6 4}$ \\
rs7975232 & CPS & C & A & 0.36 & 0.41 & 1.21 & 0.72 & 2.03 & $\mathbf{0 . 4 5}$ & $\mathbf{0 . 0 1 6}$ \\
rs4588 & SLE & T & G & 0.23 & 0.27 & 1.24 & 0.77 & 2 & 0.42 & 0.48 \\
rs4588 & RA & T & G & 0.23 & 0.22 & 0.93 & 0.56 & 1.53 & 0.81 & 0.84 \\
rs4588 & SS & T & G & 0.23 & 0.21 & 0.89 & 0.47 & 1.65 & 0.77 & 0.76 \\
rs4588 & CPS & T & G & 0.23 & 0.29 & 1.37 & 0.76 & 2.43 & 0.26 & 0.53 \\
\end{tabular}

Conclusion: There is evidence of nominal association between VDR SNPs: rs731236 (in SLE and CPS), and rs7975232 (in SS and CPS) and the presence of IMIDs disease in Paraguayan patients.

Disclosure of Interests: None declared

DOI: 10.1136/annrheumdis-2021-eular.4299

\section{Adaptive immunity ( $T$ cells and B cells) in rheu- matic diseases}

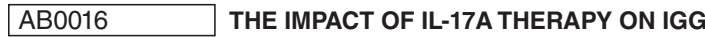 SIALYLATION IN HUMANS}

R. Pfeifle ${ }^{1,2}$, J. Kittler ${ }^{1}$, M. Wuhrer ${ }^{3}$, G. Schett ${ }^{1}$, G. Krönke ${ }^{1} .{ }^{1}$ University Hospital Erlangen, Department of Internal Medicine 3 and Institute of Clinical Immunology, Erlangen, Germany; ${ }^{2}$ Friedrich-Alexander University, NikolausFiebiger Center of Molecular Medicine, Erlangen, Germany; ${ }^{3}$ Leiden University Medical Center, Department of Rheumatology, Leiden, Netherlands

Background: Rheumatoid arthritis (RA) is characterized by autoreactive $B$ and T cells. Autoantibodies are a hallmark of RA and contribute to synovial inflammation. We have recently demonstrated that Th17 cells suppress the enzyme ST6 a-galactoside b-2,6-sialyltransferase (ST6GAL1) in developing plasma cells. Thereby, Th17 cells regulate the degree of autoantibody sialylation leading to the increased inflammatory activity of autoantibodies. These events correlate with the onset of RA, arguing for a crucial role of the IL-23/ Th17 axis during the transition of asymptomatic autoimmunity into active RA. Therefore, treatment against the IL-23/TH17-axis might present an attractive therapeutic approach to halt or delay RA's onset. However, the effects of Th17 cytokines like IL-17 on IgG glycosylation in humans are so far poorly studied. Objectives: To explore whether anti-IL17A treatment can inhibit pro-inflammatory IgG glycosylation patterns in humans.

Methods: Total IgG from patient cohorts suffering from psoriatic arthritis (PsA) treated with Secukinumab (anti-IL-17 blockade, $\mathrm{n}=26$ ) or Ustekinumab (anti-IL12/23 blockade, $n=14$ ) was compared with patients treated with anti-TNFa blockade as a control $(n=20)$. The cohorts were age- and sex-matched and included patients being on therapy for at least six months. Total IgG was isolated using Protein $G$ columns, and IgG glycopeptides of IgG1, IgG2, and IgG4 were analyzed using the LC-MS technique. The effect of IL-17 depletion on IgG glycosylation was analyzed in psoriatic arthritis patients who have been treated with secukinumab for at least six months. Furthermore, in a longitudinal approach, IgG1, IgG2, and IgG4 glycosylation were analyzed from samples, isolated before the beginning of anti-IL-17 blockade and after at least six months of therapy $(n=16)$.

Results: Cross-sectional comparison of cohorts treated with Ustekinumab, Sekukinumab, and anti-TNFa therapy did not show any significant differences in sialylation, galactosylation, or fucosylation of $\lg$ G1 and $\lg G 2$. IgG4 from antiTNFa treated patients displayed a small increase of sialylation when compared to the Ustekinumab treated cohort.

Longitudinal analyses, however, showed that IL-17A blockade during Secukinumab therapy caused a significant increase of sialic acid-rich IgG glycoforms on lgG1 IgG2 IgG4 patients, while the galactosylation, fucosylation remained unaffected.

Conclusion: This data indicates that IL-17A blockade specifically affects IgG sialylation, while other Fc-glycan modifications remain unaltered. This data confirms our recent findings in mice, where cytokines of the IL-23/Th17 axis specifically induce the production of hypo-sialylated, proinflammatory autoantibodies in 